Cahiers d'études italiennes

\title{
La figure du juif dans La Storia d'Elsa Morante
}

\section{Sophie Nezri-Dufour}

\section{OpenEdition \\ Journals}

Édition électronique

URL : http://journals.openedition.org/cei/911

DOI : $10.4000 /$ cei.911

ISSN : 2260-779X

Éditeur

UGA Éditions/Université Grenoble Alpes

\section{Édition imprimée}

Date de publication : 15 mai 2008

Pagination : 65-74

ISBN : 978-2-84310-121-2

ISSN : $1770-9571$

Référence électronique

Sophie Nezri-Dufour, "La figure du juif dans La Storia d'Elsa Morante », Cahiers d'études italiennes [En ligne], 7 | 2008, mis en ligne le 15 novembre 2009, consulté le 20 avril 2019. URL : http:// journals.openedition.org/cei/911; DOI : 10.4000/cei.911 


\title{
LA FIGURE DU JUIF \\ DANS LA STORIA D'ELSA MORANTE
}

\author{
Sophie Nezri-Dufour \\ Université de Provence, Aix-Marseille 1
}

À bien considérer le roman d'Elsa Morante, La Storia, il apparaît que la tragédie juive est au centre d'une réflexion qui englobe un grand nombre de thématiques développées dans le récit. Dans ce roman, Morante s'attache à dépeindre et à dénoncer le processus par lequel la différence culturelle et sociale des juifs est transformée en faute et en impureté. Les juifs sont, dans son propos, les représentants paradigmatiques des parias, des exclus, de ceux qui dérangent et inquiètent, que l'on désire voir disparaître, et que l'Histoire, monstre destructeur, d'après l'auteur, n'hésite pas à broyer.

La période des lois raciales reflète en effet une véritable névrose vis-àvis de l'Autre, l'Autre qui menace, par ses quelques différences, l'identité collective. Pour démonter et dénoncer cet état d'esprit, Morante cite dans son roman des articles entiers émanant des lois antisémites élaborées par les pseudo-scientifiques italiens de l'époque qui furent chargés de définir, et c'est ce qu'ils firent avec zèle, mais sans aucun fondement scientifique, l'identité raciale du juif ${ }^{*}$.

Ces décrets d'exclusion montrent bien à quel point l'altérité du juif posait problème, car était légalement considéré juif celui qui, par exemple, avait un parent juif et un autre étranger, comme si la judéité était liée à un univers dès le départ inconnu, autre, intrinsèquement différent et allogène, s'opposant par définition à l'idée de cohésion nationale: dans ces lois antisémites italiennes, qui furent d'ailleurs moins bien appliquées mais plus pointilleuses que les lois nazies ${ }^{2}$, l'Italien juif était par essence un étranger, aussi différent d'un Italien que l'aurait été un Français ou un Allemand. 
Dans une logique imparable s'inspirant de l'idéologie nazie, le juif, devenant un étranger dans son propre pays, sortait de facto de l'univers des gens normaux, de ceux qui forment une collectivité soudée, apparemment privilégiée, constituée d'êtres supérieurs, en l'occurence les aryens, comme l'étaient devenus les Italiens de l'époque. A ce propos, Morante écrit:

Gli ebrei erano diversi non solo perché ebrei, ma anche perché non ariani. E chi erano gli Ariani? A Iduzza questo termine delle Autorità suggeriva qualcosa di antico e d'alto rango, sul tipo di barone o conte. E nel suo concetto gli ebrei vennero a contrapporsi agli ariani, più o meno, come i plebei ai patrizi [...]. Però, evidentemente, i non ariani, per l'Autorità, erano i plebei dei plebei! Per esempio, il garzone del panettiere, plebeo di classe, di fronte a un ebreo valeva un patrizio, in quanto ariano! E se già i plebei nell'ordine sociale erano una rogna, i plebei dei plebei dovevano essere una lebbra! (p. 57)

On voit bien là comment de l'exclusion civique, on glisse à l'exclusion sociale, et à la marginalisation. Le juif, parce que différent, devient un paria, un individu impur et infâme, coupable d'une faute mystérieuse, mais qui devient bien réelle et à laquelle une partie de la collectivité nationale va finir par croire, puisqu'elle est érigée en loi. Ainsi, Ida, en tant que demi-juive, sent-elle peser sur elle " il marchio dei reprobi e degli impuri [...]. Si sentiva in colpa, come un'abusiva e una falsaria " (p. 58).

Les juifs, différents, sont ainsi catalogués et exposés à un mépris officialisé, légitime, légal et légalisé, qui engendre une logique que plus personne n'essaie de remettre en cause: "Secondo la legge dei tedeschi gli ebrei erano pidocchi, e andavano tutti sterminati " (p. 61). Ce n'est pas pour rien que Morante parlera, en évoquant la rafle du ghetto de Rome, d'un quartier vidé « di tutta la carne giudia » (p. 237).

Dans ce processus d'exclusion de l'Autre, que Morante décrypte à travers de nombreuses informations historiques, la collectivité italienne semble peu à peu prendre ses distances avec la communauté juive. Désormais deux mondes différents, imperméables, se côtoient. Lorsque l'auteur décrit en effet la situation des juifs du ghetto enfermés dans les wagons à bestiaux de la Stazione Tiburtina, on se trouve face à un drame où plane une totale irréalité due en grande partie à une indifférence générale de la population. Les juifs appartiennent désormais à un autre univers, un infra-monde, pareil à celui des pestiférés: un univers qui connaît une telle tragédie qu'il en devient tabou, terrible, susceptible d'infecter ceux qui s'en approcheraient. Alors que tous les juifs ont été enfermés dans des wagons plombés, Morante décrit la gare déserte, d'apparence tranquille: l'abandon des juifs est total: «Oggi non vi si notavano militari, e soli pochi borghesi vi si aggiravano senza fretta. In quella 
tarda mattina di lunedì, l'edificio aveva un'aria abbandonata e provvisoria.» (p. 242)

Bien que l'innommable soit en train de se réaliser, la vie continue, au sein même de la gare, où les activités se poursuivent dans la plus totale sérénité. L'indifférence est absolue, effrayante, comme si deux mondes parallèles étaient en train de se côtoyer, à travers des frontières invisibles, sans qu'aucun rapport ne soit désormais plus jamais possible. Le destin des juifs semble arrêté, il est donc inutile de s'y opposer:

Quella zona della stazione appariva, attualmente, deserta e oziosa. Non c'era né movimento di treni, né traffico di merci; e le sole presenze che si scorgessero erano, di là dal limite dello scalo, distanti entro la zona della ferrovia principale, due o tre inservienti del personale ordinario, dall'apparenza tranquilla. (p. 243)

Dans ces termes exprimant volontairement la tranquillité - « senza fretta ", " ordinario ", " oziosa ", " tranquilla " - on relève un contraste extrême entre la tragédie des juifs, entassés dans les trains de la mort, et le caractère tranquille, anodin, ordinaire de la vie de ceux qui ne sont pas juifs, qui ne sont pas différents.

Ce contraste ressort d'autant plus à travers un épisode historique authentique, avéré, un épisode qui met en scène un des personnages les plus dramatiques du roman, la Signora Di Segni, d'origine juive, qui n'a pas été raflée mais qui veut rejoindre sa famille enfermée dans les wagons: elle s'est rendue à la gare et, dans la plus parfaite solitude, comme nous venons de le voir, elle tente d'ouvrir la porte du wagon où se trouvent les siens. Là encore, il n'y a personne, et comme dans un cauchemar, le personnage évolue dans un désert d'horreur où la tragédie semble se dérouler sans même l'action humaine: "Essa non incontrò nessuno, salvo un macchinista solitario che mangiava da un cartoccio, vicino a una locomotiva spenta, e non le disse nulla. Forse, anche i pochi sorveglianti erano andati a mangiare. » (p. 243)

L'absence de l'humain, et de l'humanité, est frappante dans ce passage. L'horreur, l'indicible se réalisent dans le silence, l'indifférence, le calme, même si un murmure inquiétant s'impose peu à peu: c'est le bruit que font les juifs dans les trains: le murmure sourd des pestiférés, des parias, des rebus d'une société qui ne veut plus d'eux, qui les veut loin d'eux, comme on le voit dans les termes soigneusement choisis par l'écrivain, qui évoquent des lieux d'anormalité:

L'invisibile vocio si andava avvicinando e cresceva, anche se, in qualche modo, suonava inaccessibile quasi venisse da un luogo isolato e contaminato. Richiamava insieme certi clamori degli asili, dei lazzaretti e dei reclusorii : però tutti rimescolati alla rinfusa, come frantumi buttati dentro la stessa macchina [...]. In quel momento non c'era nessuno di guardia al treno. (p. 243-244) 
Alors que la Signora Di Segni a réussi à localiser le wagon où se trouvent son mari et ses enfants, la voix qui en sort est décrite par Morante comme " atona, estranea, come da un atroce paradiso di là da ogni recapito " (p. 244). Il semble y avoir désormais deux mondes différents, avec leur réalité propre. L'univers des déportés est définitivement devenu un monde à part: un monde où régne l'horreur, la folie, l'absurde, la déshumanisation, l'animalité:

L'interno dei carri, scottati dal sole ancora estivo, rintronava sempre di quel vocio incessante. Nel suo disordine, s'accalcavano dei vagiti, degli alterchi, delle salmodie da processione, dei parlottii senza senso, delle voci senili che chiamavano la madre; delle altre $[\ldots]$ cerimoniose e delle altre che perfino ridacchiavano. E a tratti su tutto questo si levavano dei gridi sterili agghiaccianti; oppure altri, di una fisicità bestiale. (p. 245)

L'univers des déportés est si effrayant que les cheminots italiens n'osent pas l'approcher. Invitant la Signora Di Segni à s'enfuir au plus vite, ils ont littéralement peur de s'approcher d'elle et des rails. Comme un lieu de malédiction, les trains de la déportation appartiennent déjà à l'univers de la mort. Renfermant l'horreur absolue, ils épouvantent les gens " normaux ", qui ont peur d'être contaminés par cette horreur extrême, par cette réalité cauchemardesque:

Degli uomini (facchini o impiegati) si agitavano a distanza verso di lei, sollecitandola coi gesti. Però, non si avvicinavano al treno. Sembravano, anzi, evitarlo, come una stanza funebre o appestata. (p. 246)

Comme on le voit bien dans ces différents passages, Morante a voulu montrer que la déportation des juifs se déroule dans un climat d'indifférence et d'irréalité totale. On se trouve face à un pouvoir sans visage, qui anéantit un peuple silencieusement, et en parfaite impunité: parce que l'humanité est totalement absente de cet acte génocidaire encore inédit, à la fois absurde et totalement rationalisé, sa réalisation s'enveloppe d'une dimension irréelle et hors-norme qui semble plonger la grande majorité de la population dans la perplexité et l'apathie.

Face à la déshumanisation des juifs, provoquée par le traitement qu'on leur inflige, l'image de leur déchéance semble s'imposer peu à peu comme une réalité logique: si les juifs sont traités comme des parias de la société dont il faut se débarrasser, c'est sans doute parce que ce sont des parias et des rebuts. L'ignominie nazi-fasciste doit certainement avoir une justification! Si l'on traite les juifs comme des sous-hommes, c'est sans doute parce que ce sont des sous-hommes. L'effet (la déshumanisation) se transforme ainsi en cause, qui permet de justifier l'injustifiable. Morante précisera d'ailleurs, que, quelques jours seulement après la rafle du ghetto 
" degli ebrei, e della loro sorte, già non si parlava più » (p. 249).

Et lorsque, aux lendemains de la guerre, les quelques survivants reviendront, ils continueront à appartenir à ce monde atroce de l'innommable, à cet univers effrayant qui les rendra à leur tour, dans la plus grande injustice, innommables et effrayants car infectés par l'horreur. Morante écrira à ce sujet :

Nessuno voleva ascoltare i loro racconti : c'era chi se ne distraeva fin dal principio, e chi li interrompeva prontamente con un pretesto, o chi addirittura li scansava ridacchiando, quasi a dirgli: "Fratello, ti compatisco, ma in questo momento ho altro da fare". [...] I giudii [...] erano figure spettrali [...] impossibili perfino alla comune simpatia. La gente voleva rimuoverli dalle proprie giornate come dalle famiglie normali si rimuove la presenza dei pazzi, o dei morti. (p. 377)

Pour exprimer ce caractère hors-norme de la persécution antisémite, Morante fait des choix littéraires très précis: bien que les données historiques concernant l'exclusion des juifs ne manquent pas, l'auteur utilise également un discours où une vision presque onirique domine, à partir d'une description du drame où les images utilisées côtoient le registre épico-tragique, ce qui ne va pas sans déformer parfois, nous allons le voir, la réalité.

La différence du juif est considérée comme une infamie, une faute, une tare, non seulement par les autorités de l'époque, et une partie de la population, mais également par les héroïnes du roman, Nora et Ida, qui semblent croire à l'existence d'un destin hostile qui s'acharnerait tragiquement sur les juifs. Nora considère en effet son identité juive comme une malédiction, " $[\mathrm{da}]$ camuffare ». Ses " occulte discendenze razziali » sont celles, d'après ce qu'elle dit,

[di un] popolo predestinato dall'eternità all'odio vendicativo di tutti gli altri popoli ; [...] la persecuzione si accanirà sempre su di loro, pure attraverso tregue apparenti, riproducendosi sempre in eterno, secondo il loro destino prescritto. (p. 22, 24)

Le drame juif tel que le considère Nora, et plus tard Ida, mais surtout Elsa Morante elle-même, acquiert une dimension mythique plus qu'historique, dans le sens où la persécution antisémite est caractérisée par une absence de progression temporelle. La fatalité, la malédiction s'acharnent sur le peuple juif broyé par une Histoire cyclique et dévastatrice, dénuée de l'idée de progrès. Chez Morante, tout s'est déjà passé, et tout doit se passer une nouvelle fois: le juif, victime d'une société qui ne supporte pas l'Autre, l'individu différent, est ainsi à la merci de forces lointaines et maléfiques, plus qu'historiques, qui s'acharnent sur lui. 
Chez Morante, le juif acquiert alors une dimension mythico-religieuse qui en fait une victime sacrificielle d'un destin hostile et cruel où le temps n'est plus linéaire mais cyclique. D'où la vision de la scène de la Stazione Tiburtina, hallucinante, où le temps semble s'être suspendu. Par là, Morante semble vouloir nous faire pénétrer dans l'atemporalité de la tragédie juive: l'auteur arrête le temps, pour mieux mettre en évidence l'exemplarité du moment. Le lecteur pénètre alors dans une dimension absolue, où la réalité juive échappe aux données temporelles et spatiales. L'action se déroule à un autre niveau, où le passé coexiste avec le présent, où le drame juif devient atemporel ${ }^{3}$.

L'individu juif se perd dans la collectivité qui est la sienne, le présent se confond avec le passé, et les différentes individualités ne forment plus qu'une seule et même entité souffrante. Ainsi, Ida, demi-juive, face aux plaintes des déportés, se sent-elle engloutie elle-même dans une réalité dont elle sait qu'elle fait partie, et dans laquelle elle se perd, fascinée et comme rattrapée par une mémoire sacrée et cyclique:

Ida riconosceva questo coro confuso [...] tutto questo misero vocio dei carri la adescava con una dolcezza struggente, per una memoria continua che non le tornava dai tempi, ma da un altro canale [...]. Era un punto di riposo che la tirava in basso, nella tana promiscua di un' unica famiglia sterminata. » (p. 245)

"Si sentiva invasa da una debolezza estrema [...]. Però, si lasciava a questa debolezza del suo corpo come all'ultima dolcezza possibile, che la faceva smarrire in quella folla, mescolata con altri sudori. (p. 246)

Mais cette circularité temporelle efface par là-même l'identité individuelle puisque ce qui devient important, mythique, sacré, c'est le destin collectif. Or, cet effacement de l'identité est une limite dans l'écriture de Morante qui désire pourtant redonner vie à ce que vécurent les victimes de la rafle du ghetto. D'où une vision du juif où domine l'empathie certaine de l'écrivain, mais qui est aussi cristallisée dans un univers qui pérennise une tragédie collective où l'individu n'existe pas en tant que tel: Morante insiste en effet sur le destin qui semble déjà écrit de la communauté juive à travers l'Histoire.

Chaque individu perd dès lors son humanité, ses particularités, et fait partie d'une réalité dépeinte selon des schémas parfois un peu arrêtés et stéréotypés. Lorsque l'auteur évoque le ghetto, la représentation qui en est faite est, à certains moments, proche de la caricature et du misérabilisme, ceci, malgré une sympathie certaine pour le peuple juif. Morante utilise en effet des termes et des diminutifs qui offrent des juifs l'image d'un peuple attachant mais figé dans des gestes et des attitudes qui semblent intemporels, marqués par une condition sociale et historique sans appel, qui ne 
semble pas connaître de progression ni d'évolution. Et même si elle dépeint quelques scènes avec le réalisme des estampes, les personnages n'en restent pas moins sans véritable visage, comme ceux qu'elle appelle " [gli] ebreucci » ou " certi tipi di vecchi, dagli occhi mezzi spenti e dalla bocca sigillata " (p. 58).

Quant à Ezechiele, la sage-femme qui porte secours à Ida au moment de son accouchement, et dont le nom indique bien, là encore, une stylisation voulue du juif, elle offre, bien que plus humanisée que les autres juifs, les traits de la caricature du juif à l'époque:

Nei sopraccigli folti, nel naso robusto e arcuato, nei grossi piedi e nella grandezza del passi, e perfino nel modo di portare il suo berrettuccio bianco di cotone sui capelli grigi e riccioluti, ricordava una stampa del profeta Ezechiele. (p. 93)

Cette stylisation des juifs revient également lorsqu'il est question de l'ensemble de la population juive du ghetto:

Da quando il vecchio quartiere [del ghetto] era stato risanato e le muraglie abbattute, il suo popolo non aveva fatto che moltiplicarsi ; e adesso, in quelle solite quattro straducce e due piazzette, ci si arrangiavano a stare a migliaia. C'erano molte centinaia di pupetti e ragazzini, per lo più riccetti, con gli occhi vispi [...]. Regnava una incredulità ingenua e fiduciosa [...]. Le poverette donne indaffarate opponevano, per lo più, una spensieratezza evasiva, oppure una rassegnazione reticente. Tante notizie [sui nazisti] erano invenzioni della propaganda. (p. 59)

Avec des mots choisis, dont des diminutifs condescendants, Morante parle en effet du fait que " i più vivevano ancora, in certo modo, nella preistoria. E così dunque non deve stupire troppo la semplice ignoranza di certe infime donnette ebree» (p. 91).

Malgré sa grande empathie, Morante n'attribue pas la moindre étincelle de conscience historique aux juifs. Humanité archaïque, glorifiée certes, ceux-ci ne forment qu'une entité sans la moindre liberté d'action ni de pensée. Cela apparaît de manière frappante dans la description des quelques déportés de la Stazione Tiburtina, que nous évoquions auparavant. Ils sont dépeints à travers une série de métonymies qui leur retirent toute humanité, toute conscience, toute personnalité. Seul quelques traits saillants, redondants, les caractérisent. Ainsi, un enfant qui cherche à voir ce qui se passe en-dehors du wagon est décrit comme " una testolina irsuta, due occhietti neri » (p. 246). Un vieillard qui désire laisser un message est dépeint par le biais de sa " povera testa calva con occhi intenti che parevano malati, [con] una mano [che] si sporse a gettare un foglietto " (p. 247). Le mari de la Signora di Segni est lui aussi dépeint partiellement, comme si son corps avait perdu son intégrité, sans doute parce qu'il n'appartient déjà plus au monde des vivants: "Si vedevano i suoi occhiali 
tralucere fra il buio retrostante, sul suo naso macilento, e le sue mani minute aggrappate ai ferri ${ }^{4} »$ (p. 244).

Les nazis, dans la rapide description qui en est faite, ont au contraire un visage humain. Ils sont aryens, ils font partie de la normalité: ils ont donc une réalité sociale:

Il loro aspetto era normale, inalterato come quello dei soliti camionisti del Comune che caricavano a questo transito dello Scalo i loro trasporti di carne. Le loro facce pulite, e rosa di salute, erano comuni e stolide. (p. 247)

On comprendra que la description volontairement métonymique des déportés est destinée à dépeindre la progressive déshumanisation à laquelle ils sont destinés. Mais leur manque de dignité et de conscience devient, dans cette peinture quelque peu pathétique, trop criant, et une ambiguité peut s'installer. Les juifs n'ont par exemple jamais de regard, et leurs yeux ne sont jamais décrits, comme c'est au contraire le cas pour Useppe, par exemple, dont l'intelligence et l'humanité, la personnalité et la vitalité, résident dans les yeux.

À travers les yeux se crée et s'élabore en effet une interférence avec la réalité, la compréhension des choses, la réaction, ce que Morante refuse à ses personnages juifs. Ces derniers sont par contre souvent comparés aux fous ou aux animaux, pour lesquels l'écrivain a une immense tendresse, mais qui demeurent dans un monde dépourvu de liberté, de dignité et de conscience sociale, historique et politique. L'animalité chez Morante est en effet la métaphore d'une existence qui n'appartient pas à l'Histoire, ou qui fuit l'Histoire, car celle-ci est trop atroce. L'animalité devient alors synonyme d'archaïsme, dans le sens de régression, d'irrationnel, de fuite du réel. Or, si les juifs sont si souvent comparés à des animaux, c'est parce qu'ils sont enfermés dans un monde où l'individu libre et pensant n'a plus place; donc, comme les animaux, ils sont écartés de la vie sociale. Ainsi, le veau de la Stazione Tiburtina, pourvu d'une étrange médaille et destiné à un sort qu'il ignore encore, l'abattoir sans aucun doute, est-il une préfiguration du sort des juifs:

Dal collo, per una cordicella, gli pendeva una medagliuccia, all'apparenza di cartone, sulla quale forse era segnata l'ultima tappa del suo viaggio. Di questa, al viaggiatore non s'era data nessuna notizia; ma nei suoi occhi larghi e bagnati s'indovinava una prescienza oscura. (p. 125)

De même, les chats que nourrit Vilma, cette Cassandre juive qui annonce l'innommable sans que personne ne la croie jamais, représentent également le destin des habitants juifs du ghetto: 
Capitava sempre d'incontrare Vilma, avvilita perché di giorno in giorno, la raccolta degli avanzi [per i gatti] si rendeva più difficile, e inoltre ogni volta aumentava, fra i suoi gatti dei ruderi, il numero degli assenti all'appello. Essa li conosceva uno per uno, e se ne informava in giro con una misera voce sconsolata: "Non s'è più visto lo Zoppetto? E Casanova? E quello senza un occhio..." (p. 90)

Dans la même logique, Blitz, le chien d'Useppe, destiné à mourir injustement, est une autre allégorie du juif. Ce n'est pas innocent si Morante le décrit comme un chien de " razza bastarda ", " di razza stellata ", avec un " bel disegno di stella " " nel mezzo della pancia ». "Questa era l'unica sua bellezza e specialità»(p. 104).

Quant à la Stazione Tiburtina qui renferme les juifs en partance pour Auschwitz, elle se transforme rapidement, dans la vision hallucinatoire d'Ida, l'héroïne, en une étable. Comme le ghetto qu'Ida considérait comme une "stalla materna, calda di respiri animali » (p. 238). Comparée aussi à " una tana » (p. 245), la gare est remplie du "grido degli animali ammucchiati nei trasporti » (p. 243). L'atmosphère aussi, caractérisée par un " orrendo brusio " (p. 243), est décrite comme étant " di una fisicità bestiale » (p. 245). La Signora Di Segni, dans son désespoir, est quant à elle " come una bestia » (p. 242), " minacciosa e inferocita " (p. 244), parce qu'on lui refuse l'entrée aux enfers des trains.

Plus conventionnelle, mais non moins stéréotypée et caricaturale sera la description des déportés romains arrivés à Auschwitz. Dans sa méconnaissance de la perversité de l'organisation nazie, Morante les comparera, par la bouche d'un personnage anonyme, un déporté survivant, " agli animali segnati, che si affidano docili al recinto del macello, facendosi caldo coi fiati l'uno all'altro » (p. 310-311). Concrètement, qu'auraient-ils pu faire? On sait aujourd'hui que l'organisation nazie empêchait tout acte de rébellion, et qu'à l'arrivée dans les camps, il était impossible, et inutile, de se révolter ou de s'enfuir.

Et c'est là que l'on note certaines limites dans la description du juif, chez Morante. Malgré sa sympathie évidente pour cette communauté, malgré son travail de mémoire exemplaire, elle ne peut s'empêcher de tomber parfois dans certains travers, influencés par des stéréotypes, des caricatures, des imageries présents depuis longtemps dans la littérature. En ce sens, elle-même démontre parfois une difficulté à intégrer l'altérité juive.

Nous n'oublierons pourtant pas que Morante fut parmi les rares écrivains, avec Giacomo Debenedetti ${ }^{5}$, à décrire la rafle tragique des juifs du ghetto de Rome. À ce propos, Giorgio Romano, dans son étude Ebrei nella letteratura, écrira: 


\section{SOPHIE NEZRI-DUfOUR}

C'est une importance particulière que revêt dans notre démonstration le roman d'Elsa Morante, La Storia, qui nous paraît être l'une des œuvres les plus extraordinaires de la littérature italienne contemporaine, où les vicissitudes des juifs d'Italie durant la Seconde Guerre Mondiale trouvent une analyse compréhensive et attentive, qui mérite d'être rappelée, dans le cadre de l'Histoire allant de 1941 à 1947. Le livre $[\ldots]$ restera probablement une des œuvres les plus significatives de notre temps et pas seulement dans la perspective qui nous intéresse. Ses protagonistes, juifs et non-juifs, la description du ghetto de Rome, l'empathie pour les persécutés, et même pour les animaux, dictent à Elsa Morante des pages inoubliables, des observations subtiles et profondes ${ }^{6}$.

\section{Notes}

1. E. Morante, La Storia [1971], Torino, Einaudi, 1995, p. 53-54. Désormais, pour les citations de ce roman, nous renverrons directement au numéro de page.

2 Voir M. Sarfatti, Gli ebrei nell'Italia fascista. Vicende, identità, persecuzione, Torino, Einaudi, 2000.

3 C. d'Angeli, «Il paradiso nella storia ", in Studi Novecenteschi, Pisa, Giardini, XXI, n 47-48, giugno-dicembre 1994, p. 221.

4 Comme dans une longue complainte, ou litanie, d'autre juifs sont décrits, symboliquement, à travers l'évocation lancinante de ces terribles " due mani aggrappate o un paio d'occhi fissi » (Ibidem, p. 244).

5 G. Debenedetti, 16 ottobre 1943, Milano, Il Saggiatore, 1959.

6 G. Romano, Ebrei nella letteratura, Roma, Carucci, 1979, p. 27. Nous traduisons. 